\title{
Graphene/Polypyrrole Nanocomposite as Electrochemical Supercapacitor Electrode: Electrochemical Impedance Studies
}

\author{
Punya A. Basnayaka ${ }^{1,2}$, Manoj K. Ram ${ }^{2,3}$, Lee Stefanakos ${ }^{2,4}$, Ashok Kumar ${ }^{1,2}$ \\ ${ }^{1}$ Department of Mechanical Engineering, University of South Florida, Tampa, USA \\ ${ }^{2}$ Clean Energy Research Center, University of South Florida, Tampa, USA \\ ${ }^{3}$ Nanotechnology Research and Education Center, University of South Florida, Tampa, USA \\ ${ }^{4}$ Department of Electrical Engineering, University of South Florida, Tampa, USA \\ Email: pbasnaya@mail.usf.edu,mkram@usf.edu, stefanak@eng.usf.edu,kumar@usf.edu \\ Received February 26, 2013; revised March 25, 2013; accepted April 17, 2013
}

Copyright (C) 2013 Punya A. Basnayaka et al. This is an open access article distributed under the Creative Commons Attribution License, which permits unrestricted use, distribution, and reproduction in any medium, provided the original work is properly cited.

\begin{abstract}
Graphene-Polypyrrole (G/PPy) was synthesized by chemical oxidative polymerization method, and electrochemical impedance spectroscopy (EIS) analysis was employed to study the frequency response characteristics of supercapacitors based on G/PPy conducting polymer nanocomposite. It is found that a uniform G/PPy nanocomposite is formed with polypyrrole (PPy) being homogeneously surrounded by graphene nanosheets. The porous structure allowed electrolyte and ions diffusion in synthesized G/PPy nanocomposite. The metallic conductivity of Graphene-polypyrrole exhibited higher knee frequency at $125 \mathrm{~Hz}$ than the knee frequency of pristine PPy at $36 \mathrm{~Hz}$. The high knee frequency of G/PPy supercapacitor is indicative of high power application and long cycle life. The G/PPy nanocomposite based supercapacitor with $1 \mathrm{M} \mathrm{H}_{2} \mathrm{SO}_{4}$ as the electrolyte showed specific capacitances of $270 \mathrm{~F} / \mathrm{g}$ at $0.1 \mathrm{~Hz}$ and $112 \mathrm{~F} / \mathrm{g}$ at $125 \mathrm{~Hz}$.
\end{abstract}

Keywords: Capacitance; Graphene; Conducting Polymers; Equivalent Circuit; Time Constant

\section{Introduction}

Supercapacitors, intermediate power and energy sources between dielectric capacitors and batteries, have emerged as an important energy storage technology due to higher power delivery, high cyclability and long life time [1-3]. They find application in an uninterruptible power sources in banking centers, hospitals, airport control towers, cell phone towers, hybrid electric vehicles, memory protection in CMOS, logic circuits, VCRs, CD players and PCs, etc. [1]. Supercapacitors are efficient energy storage devices, but they do have limitations in both cost and performance [1].

Carbon materials (e.g., activated carbon, carbon nanotubes (CNTs), graphene $(\mathrm{G})$ ), transition metal oxides (e.g., $\mathrm{RuO}_{2}, \mathrm{MnO}_{2}$ ), conducting polymers (e.g., polyaniline (PANI), polypyrrole (PPy), and polythiophenes (PThs)) and their nanocomposites are major material categories found in supercapacitor electrode materials [413]. The nanocomposite materials have been extensively studied for supercapacitor applications due to their high specific capacitance and high stability [14,15]. Recently, we studied the supercapacitor behavior of synthesized GPANI, G-PTh and G-polyethylenedioxythiophene (PEDOT) conducting nanocomposite materials [16-19]. The G-CPs nanocomposite has shown higher conductivity as well as specific capacitance than the parent material, either conducting polymer or graphene structure. The G/ PPy has been shown to perform as an excellent supercapacitor electrode material with specific capacitance of $267 \mathrm{~F} / \mathrm{g}, 482 \mathrm{~F} / \mathrm{g}, 409 \mathrm{~F} / \mathrm{g}$ in a three-electrode configuration using $\mathrm{H}_{2} \mathrm{SO}_{4}$ as electrolyte $[14,20]$. The maximum specific surface area of $98.62 \mathrm{~m}^{2} \cdot \mathrm{g}^{-1}$ has been reported for the G/PPy nanocomposite electrode. The novel nanostructure of PPy with multilayered highly electrically conductive graphene nanosheets (GNS) for supercapacitor applications have also been reported [14]. The achieved specific capacitance was reported as $164 \mathrm{~F} / \mathrm{g}$ in a two-electrode configuration using $1 \mathrm{M} \mathrm{NaCl}$ as electrolyte [21].

Activated carbon has been used commercially for high power applications with the knee frequency of $1 \mathrm{~Hz}$ [22- 
24]. Carbon nanotubes (CNTs) and graphene have shown frequency response having knee frequencies of $6 \mathrm{~Hz}-$ $100 \mathrm{~Hz}$ for carbon nanotube [22], and $360 \mathrm{~Hz}$ for graphene [25]. Although some efforts have been made on the G/CPs-based supercapacitors, it is still challenging to use these nanocomposites as supercapacitor electrode materials for high power applications.

The objectives of this manuscript are to introduce a high yield simple synthesis route of G/PPy nanocomposite with large surface area and characterize the G/PPy supercapacitor electrode based on the frequency response studies in a two-electrode system supercapacitor cell for high power applications. The G/PPy was synthesized by oxidative polymerization with a high yield of $97 \%$ with respect to the used quantities of pyrrole monomer and graphene platelets. The physical and chemical characterizations of G/PPy nanocomposites were investigated using Scanning Electron Microscopy (SEM), Raman Spectroscopy and multipoint Brunauer, Emmett and Teller (BET) surface area measurement techniques. The electrochemical impedance spectroscopy (EIS) measurements were carried out to investigate the frequency response behavior of the G/PPy as an electrode material for electrochemical supercapacitors. Finally, the electrochemical behavior was investigated by using cyclic voltammetry (CV) and galvanostatic charging/dischargeing measurement techniques, respectively.

\section{Experimental}

\subsection{Materials}

The monomer pyrrole (98\%), ferric chloride $\left(\mathrm{FeCl}_{3}\right)$, hydrochloric acid $(\mathrm{HCl})$, ethanol and methanol are all A.C.S. grade and were purchased from Sigma-Aldrich (USA). The graphene platelets (less than $10 \mathrm{~nm}$ in thickness) were purchased from Angstron Materials (USA). All other chemicals and materials were employed as purchased without any modifications unless indicated in the manuscript.

\subsection{Synthesis of G-PPy Nanocomposites}

The G-PPy nanocomposites were prepared by chemical oxidative polymerization involving monomer and graphene nanosheets in acidic media (1M HCl). The molar ratio of monomer and oxidant $\left(\mathrm{FeCl}_{3}\right)$ was kept as 1:1 for all nanocomposite material synthesis processes. The graphene to monomer weight ratio was kept as 1:1 for synthesis of all G-PPy nanocomposites. Initially, the monomer was added to $200 \mathrm{ml}$ of $1 \mathrm{M} \mathrm{HCl}$ solution, and stirred for 30 minutes before adding the graphene to the resulting solution. The graphene was added slowly and stirred for another $45 \mathrm{~min}$. A $100 \mathrm{ml}$ mixture of concentrated
$\mathrm{HCl}$ and oxidant was slowly added drop wise to the monomer solution under stirring in an ice bath at $4^{\circ} \mathrm{C}$ $5^{\circ} \mathrm{C}$ temperature. The system was kept stirred continuously for 24 hours. The solution was centrifuged for 20 minutes to precipitate the resulting nanocomposite based on PPy and graphene. The prepared nanocomposite was filtered and rinsed with methanol and deionized water. Finally, the G/PPy nanocomposite was dried at $100^{\circ} \mathrm{C}$ in an oven for $24 \mathrm{hrs}$. The PPy was synthesized by following the same route without adding graphene. The final weight of the nanocomposite and PPy were weighted in three sets of synthesis and it was found to be approximately the same. The average yield of the synthesized G/ PPy nanocomposite was measured with respect to the initial pyrrole monomer and graphene weights.

\subsection{Characterization of the G-PPy Conducting Polymer}

We used SEM and Raman spectroscope techniques to characterize the G-PPy nanocomposites. The Raman spectra of G-CP nanocomposites were recorded on a Renishaw Raman instrument by employing a $514 \mathrm{~nm}$ laser beam. Sandwich structure supercapacitor cells were fabricated with $2 \mathrm{~cm}^{2}$ area graphite substrates for all electrochemical measurements. The test electrodes (G-PPy and PPy) were prepared by mixing G-PPys with nafion and spin coated on the graphite substrate. The laboratory scale supercapacitor cell was fabricated by stacking of two G/PPy electrodes with a separator soaked in electrolyte to carry out all the electrochemical measurements. The impedance measurements are recorded on VoltaLab electrochemical workstation at a dc bias of $0 \mathrm{~V}$ with a 10 $\mathrm{mV}$ amplitude sinusoidal signal over $100 \mathrm{mHz}$ to 100 $\mathrm{kHz}$ frequency range to understand the internal resistance of the supercapacitor, and to derive the equivalent circuit model. Further, electrochemical studies of G-CPs were investigated using $\mathrm{CV}$, charging/discharging and impedance measurements. The CVs were recorded at different scan rates $(100,50,25,10$ and $5 \mathrm{mV} / \mathrm{s})$ to understand the G-CPs redox processes, and to evaluate the supercapacitor performances. All electrochemical experiments were carried out using $1 \mathrm{M} \mathrm{H}_{2} \mathrm{SO}_{4}$ electrolytic media.

\section{Results and Discussion}

The SEM image of G/PPy porous structure is shown in Figure 1(A). Figure 1(B) shows the uniform spherical build-up of PPy on top of each other with some pores throughout the film. Figure 1(C) shows the chemical structures of G-PPy which have been studied for supercapacitor applications. The CP backbone structure and graphene both provide a pathway for free charges to move through the polymer backbone chain by a sequen- 

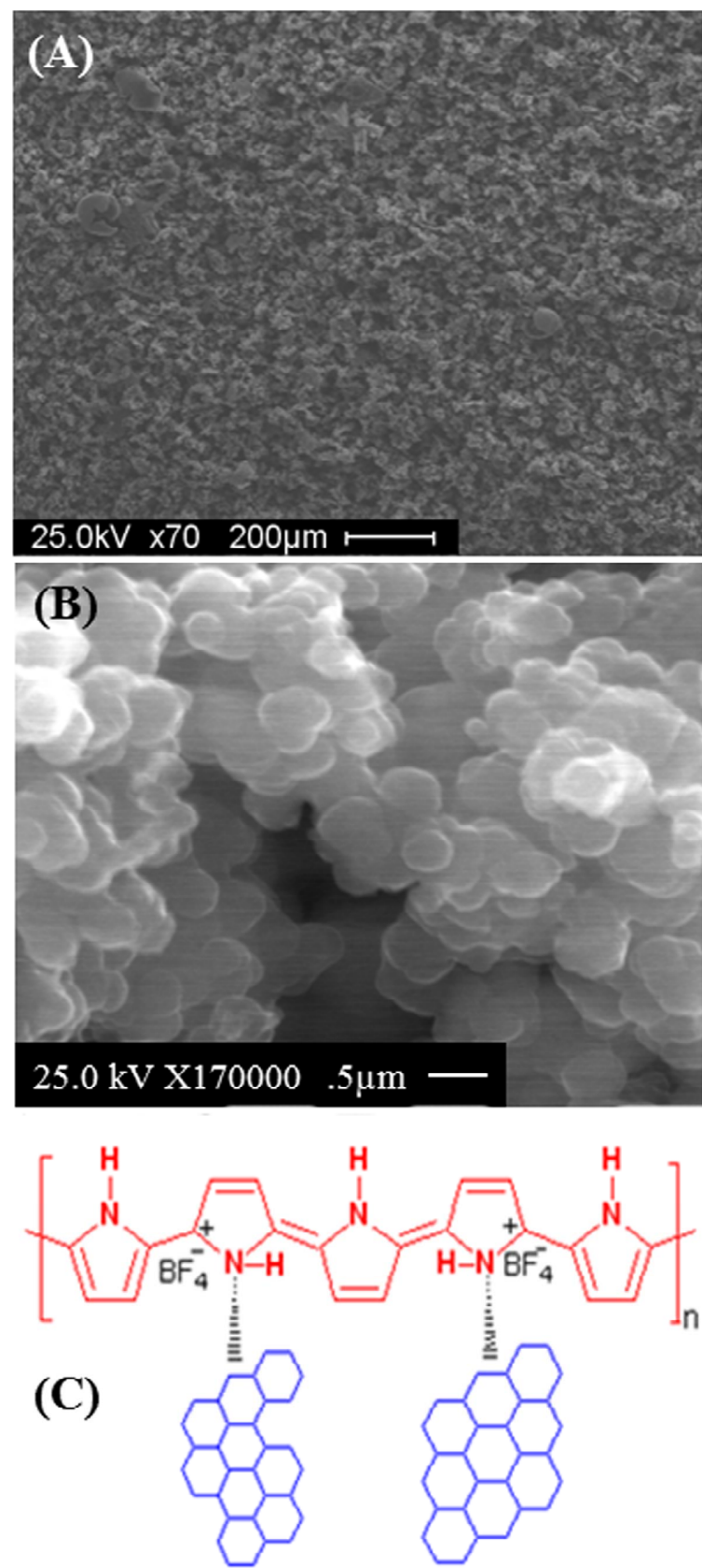

Figure 1. (A) and (B) SEM image of G/PPy electrode surface; (C) Chemical structure of G/PPy nanocomposite.

tial delocalization with $\pi$-bonds (hopping) under an applied electric field or during the doping process. The porous structure of G-PPy provides a higher surface area in contact with the electrolyte, producing a high specific capacitance with fast redox processes and high conductivity.

A BET-surface area measurement of G/PPy nanocomposite is shown in Figure 2 by plotting nitrogen adsorption and the BET surface area for G/PPy. The specific surface area of PPy and G/PPy are estimated to be $37.4 \mathrm{~m}^{2} \cdot \mathrm{g}^{-1}$ and $260.2 \mathrm{~m}^{2} \cdot \mathrm{g}^{-1}$. The increased surface area

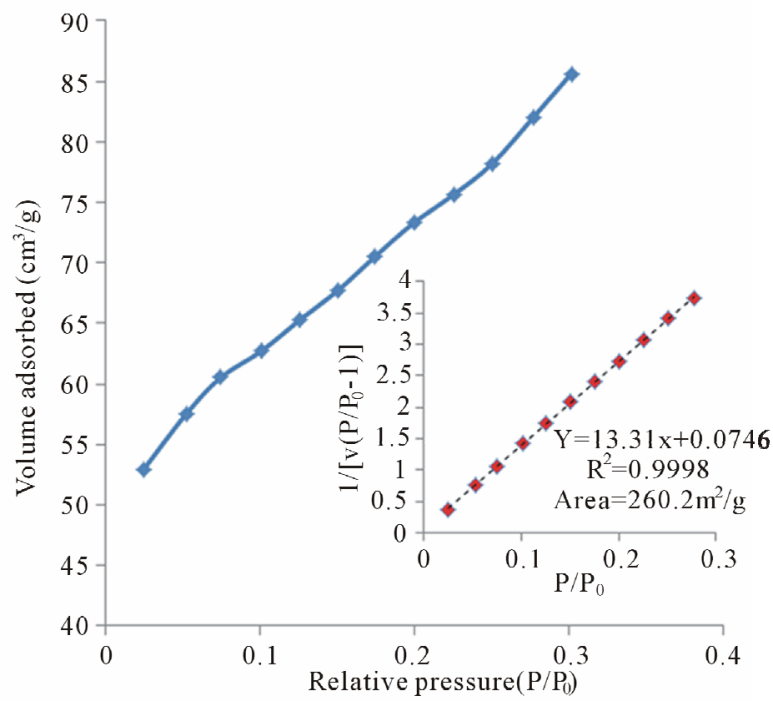

Figure 2. Plot of nitrogen adsorption and BET surface area.

of the nanocomposite increases the electrochemical activity of G/PPy nanocomposite over the PPy CP.

The G/PPy nanocomposite has shown the high weight percent yield of $97 \%$. However, it was found that the yield of PPy (81\%) was less than the yield of the G/PPy nanocomposite. A recent study on the synthesis of PPy reported a higher yield of $83.77 \%$ by using an inverted emulsion polymerization technique [26]. The calculated high yield is due to the agglomeration of PPy around the graphene. These attachments of PPy on the surface of the graphene provide the short diffusion paths and facilitate the electrochemical activities of PPy with graphene in the G/PPy nanocomposite.

The Raman spectrum of G-PPy is shown in Figure 3. The Raman peak at $1582 \mathrm{~cm}^{-1}$ is assigned to $\mathrm{C}=\mathrm{C}$ backbone stretching and the peak has been attributed to the $\mathrm{G}$ band of graphene. The peak at $1330 \mathrm{~cm}^{-1}$ corresponds to the $\mathrm{D}$ band of graphene [27]. The peak at $986 \mathrm{~cm}^{-1}$ is assigned to ring vibration of PPy, whereas bands 1073 and $1130 \mathrm{~cm}^{-1}$ are due to C-H stretching. A Raman study confirms the presence of PPy and graphene in the structure of the G/PPy nanocomposite film.

Figure 4(A) shows the measured impedance spectrum of G/PPy and PPy supercapacitors at room temperature. Figure 4(B) shows the impedance plot for the high frequency region, where the equivalent series resistance (ESR) decreases towards higher frequencies and intersects the real axis at about $5.35 \mathrm{ohm}$ for G/PPy and $5.55 \mathrm{ohm}$ for PPy.

The low ESR of G/PPy reveals a higher conductivity of the G/PPy than the PPy due to graphene incorporation in the nanocomposite material. At such high frequencies, the capacitor behaves like a simple resistor. The region with the $45^{\circ}$ slope, which is called the Warburg region, is a 
consequence of the behavior of the porous structure and is often called the distributed resistance/capacitance of G/ PPy. Finally, at low frequencies $(<125 \mathrm{~Hz}$ for G/PPy and $<36 \mathrm{~Hz}$ for PPy), the spectrum approaches a non-vertical line associated with the adsorption process, microscopic charge transfer and surface roughness. The frequency "knee" in the plot is found at $125 \mathrm{~Hz}$ for G/PPy and $36 \mathrm{~Hz}$ for PPy based electrodes. This suggests that most of the energy stored can be accessible below the frequencies of $125 \mathrm{~Hz}$ for G/PPy and $36 \mathrm{~Hz}$ for PPy. By comparison, in the literature it is reported that the knee frequency for CNT-based capacitors is about $100 \mathrm{~Hz}$ [22]. Also, for most commercially available electrochemical capacitors, especially designed for high power applications is less

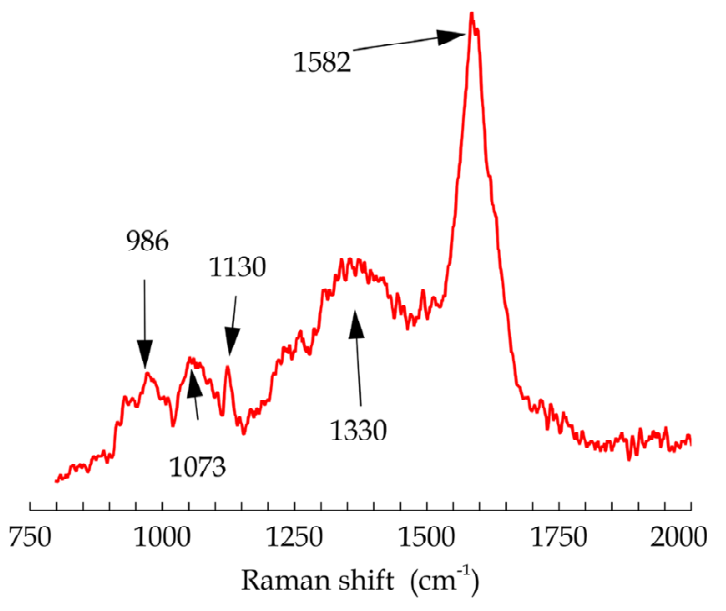

Figure 3. Raman spectra of G/PPy nanocomposite.

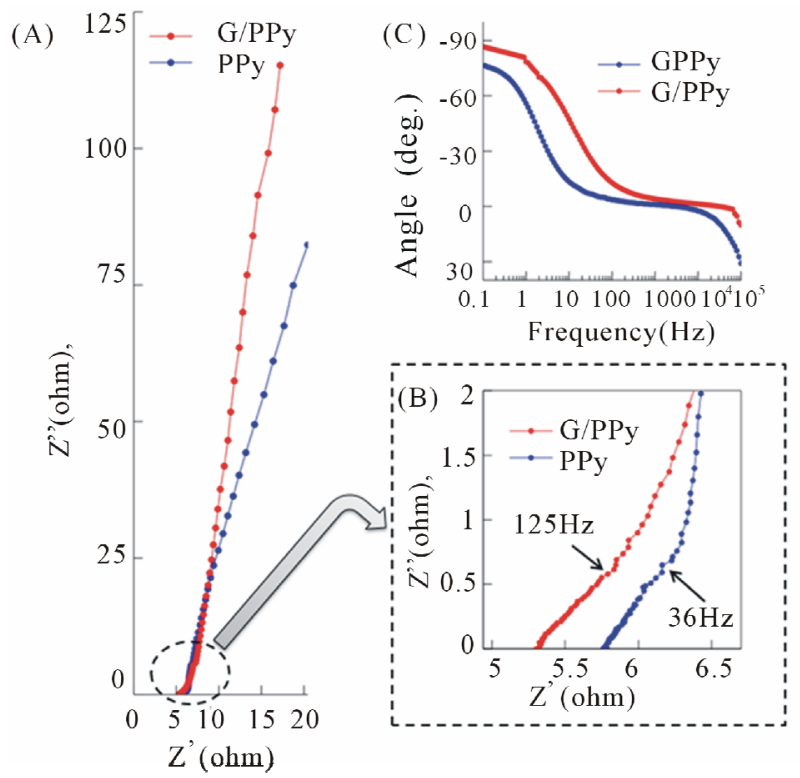

Figure 4. (A) Impedance plot over the entire frequency spectrum; (B) High-frequency impedance plot; (C) The Bode phase-angle plot. than $1 \mathrm{~Hz}$ [22-24]. Therefore the recorded knee frequency of the G/PPy supercapacitor is unique for high power applications. Figure 4(C) shows the Bode phase angle plot of G/PPy and PPy. The phase angle is very close to $-90^{\circ}$ for frequencies up to $1 \mathrm{~Hz}$ suggesting the G/PPy supercapacitor device behaves as an ideal capacitor at low frequencies [24]. On the other hand, the phase angles of the PPy supercapacitor reaches $-80^{\circ}$, suggesting that the G/PPy based supercapacitor behaves more like an ideal capacitor than the PPy.

A hypothetical electric circuit consists of parameters with well-defined electrical properties used to describe the EIS response of the G/PPy-based supercapacitor. Figure 5(C) is the circuit model and the parameters; $R_{s}$ is the electrolyte resistance in the cell, $\mathrm{R}$ is the charge transfer resistance, CPE is a constant phase angle element, and $\mathrm{Zw}$ represent the Warburg diffusion.

Figure 5(A) illustrates the EIS measurement and simulation of circuit responses with a good fit and Figure 5(B) is the EIS measurement and simulation of circuit responses for high-frequency.

The dependence of the capacitance on frequency is shown in Figure 6. The specific capacitances are calculated as $270 \mathrm{~F} / \mathrm{g}$ and $112 \mathrm{~F} / \mathrm{g}$ at frequencies 0.1 and 125 $\mathrm{Hz}$, and show better power performance using G/PPy electrode. In Figure 6, it is observed that the capacitance is fairly constant at low frequencies.

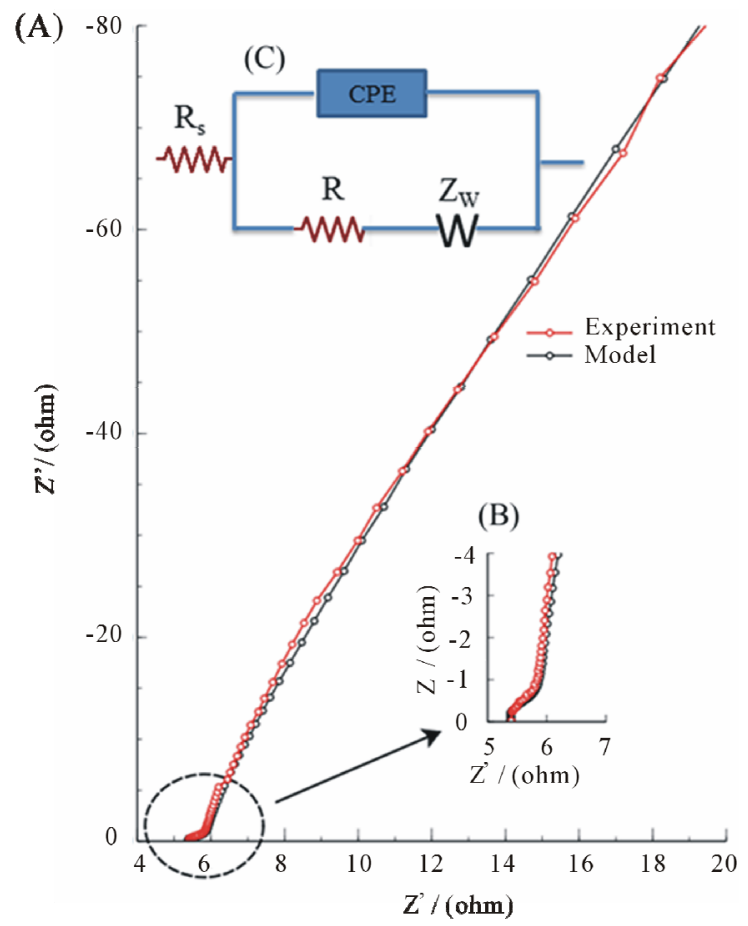

Figure 5. (A) Experimental and simulation plot of the complex-plane impedance; (B) High frequency Experimental and simulation plot of the complex-plane impedance; (C) Equivalent circuit model. 


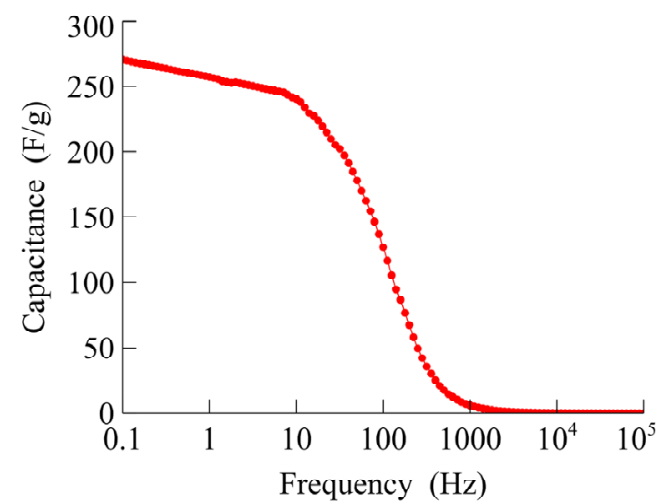

Figure 6. Capacitance dependence with frequency.

The dielectric losses in the supercapacitor due to energy dissipation by an irreversible process lead to hysteresis. The time constant $\left(\Gamma_{0}=1 / f_{0}\right)[23,28]$ was earlier described as the dielectric relaxation time constant or the supercapacitor factor of merit. The time constants are calculated to be $8 \mathrm{~ms}$ and $27 \mathrm{~ms}$ for G/PPy and PPy based supercapacitors. The difference in the time constant is due to structural changes in the electrodes of the two electrochemical capacitors.

Finally, the electrochemical behavior of G/PPy based supercapacitor is studied by $\mathrm{CV}$ measurements at different scan rates, as shown in Figure 7(A). Figure 7(B) shows the measured $\mathrm{CV}$ at $25 \mathrm{mV} / \mathrm{s}$ for the G/PPy supercapacitor, the PPy supercapacitor and only the graphite substrate without any active material on it. The G/PPy supercapacitor shows synergistic effect with respect to PPy or graphite substrate. The shape of the CVs is slightly different than ideal capacitor due to the Faradaic reaction of the conducting polymers.

Galvanostatic charge/discharge measurements were carried out at different discharge currents for a G/PPy based supercapacitor, and Figure 8 shows the charge/discharge curves. The specific capacitance of the G/PPy supercapacitor is calculated using galvanostatic discharge curves according to the Equation (1).

$$
C=i \times \Delta t /(m \times \Delta V)
$$

where, $i$ is the current, $\Delta V$ is the potential window, $\Delta t$ is the discharge time, $\mathrm{m}$ is the mass of the active material in a single electrode. The highest specific capacitance is calculated to be $256 \mathrm{~F} / \mathrm{g}$ at the discharge current density of $0.5 \mathrm{~mA} / \mathrm{g}$ for G/PPy based supercapacitor.

\section{Conclusion}

In conclusion, we have presented the frequency dependent properties of large-area G/PPy based supercapacitors in $1 \mathrm{M} \mathrm{H}_{2} \mathrm{SO}_{4}$ electrolyte, particularly for high-power applications. The knee frequency of G/PPy and PPy are found to be 125 and $36 \mathrm{~Hz}$. The addition of graphene to
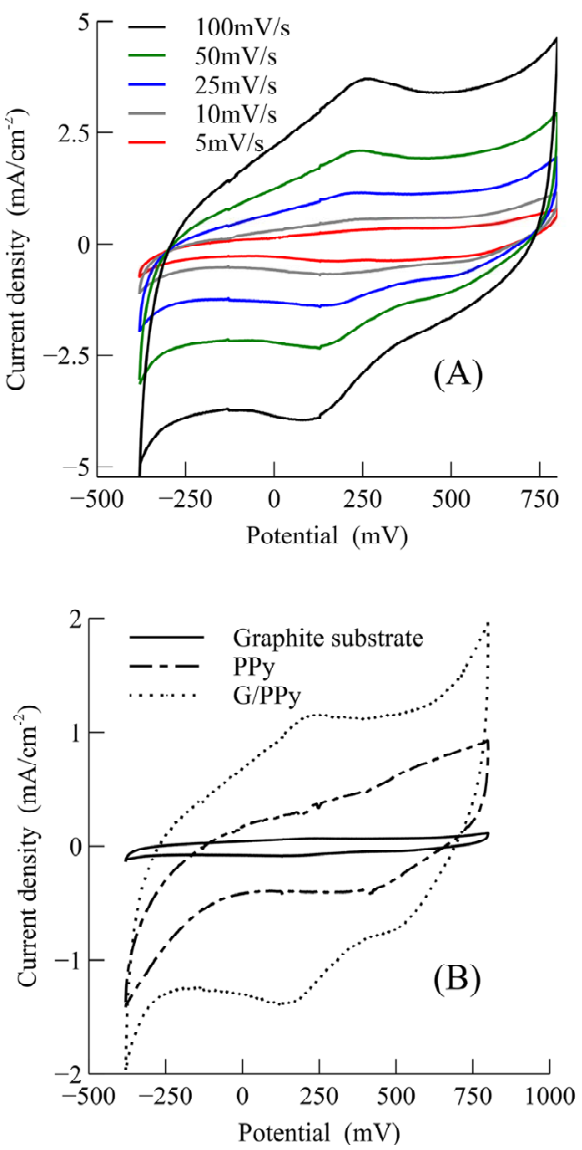

Figure 7. (A) CV of the G/PPy supercapacitor at different scan rates; (B) $\mathrm{CV}$ of the G/PPy, PPy, and graphite substrate at a scan rate of $20 \mathrm{mV} / \mathrm{s}$ in $1 \mathrm{M} \mathrm{H}_{2} \mathrm{SO}_{4}$ electrolyte.

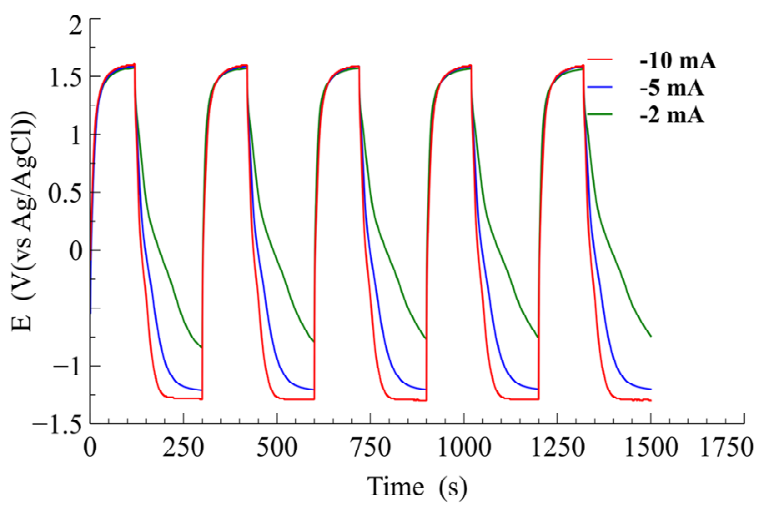

Figure 8. Charging-discharging cycles of the G-PPy supercapacitor with a $10 \mathrm{~mA}$ charging current and different discharging currents in $1 \mathrm{M} \mathrm{H}_{2} \mathrm{SO}_{4}$ electrolyte.

the nanocomposite electrode increases the knee frequency four times by reducing the internal resistance of the supercapacitor. The phase angle at the low frequency region is close to $-90^{\circ}$ for the G/PPy supercapacitor indicating a significant reduction of the internal resistance in the G-PPy nanocomposite electrode. Further, the calculated specific capacitance of $270 \mathrm{~F} / \mathrm{g}$ from EIS data is 
close to the value calculated from the gal vanostatic charging/discharging measurement technique. The electrical circuit model parameters describing the effect of the porous structure, ion diffusion in an aqueous electrolyte/ G/PPy, and charge transfer effect is presented with a simulation of the G/PPy supercapacitor with a good fit to the experimental data. The unique pore structure of the G/PPy electrodes and the excellent frequency response enables their operation for a number of power applications, and could be used for the commercial development of graphene-CP based electrodes.

\section{Acknowledgements}

We would like to acknowledge the Clean Energy Research Center (CERC) at the University of South Florida for the help in carrying out this research and Dervis Emre Demirocak for helping out in the BET surface measurements of the materials.

\section{REFERENCES}

[1] R. Kotz and M. Carlen, "Principles and Applications of Electrochemical Capacitors," Electrochimica Acta, Vol. 45, No. 15, 2000, pp. 2483-2498.

[2] B. E. Conway, "Electrochemical Supercapacitors: Scientific Fundamentals and Technological Applications," Springer, Berlin, 1999. doi:10.1007/978-1-4757-3058-6

[3] G. Wang, L. Zhang and J. Zhang, "A Review of Electrode Materials for Electrochemical Supercapacitors," Chemical Society Reviews, Vol. 41, No. 2, 2012, pp. 797-828. doi:10.1039/c1cs15060j

[4] I. H. Kim and K.-B. Kim, "Ruthenium Oxide Thin Film Electrodes for Supercapacitors," Electrochemical and Solid-State Letters, Vol. 4, No. 5, 2001, pp. A62-A64. doi:10.1149/1.1359956

[5] G. Arabale, D. Wagh, M. Kulkarni, I. Mulla, S. Vernekar, K. Vijayamohanan and A. Rao, "Enhanced Supercapacitance of Multiwalled Carbon Nanotubes Functionalized with Ruthenium Oxide," Chemical Physics Letters, Vol. 376, No. 1-2, 2003, pp. 207-213.

[6] Z. Algharaibeh, X. Liu and P. G. Pickup, "An Asymmetric Anthraquinone-Modified Carbon/Ruthenium Oxide Supercapacitor," Journal of Power Sources, Vol. 187, No. 2, 2009, pp. 640-643. doi:10.1016/j.jpowsour.2008.11.012

[7] E. Frackowiaka and F. Beguinb, "Carbon Materials for the Electrochemical Storage of Energy in Capacitors," Carbon, Vol. 39, No. 6, 2001, pp. 937-950. doi:10.1016/S0008-6223(00)00183-4

[8] H. Pan, J. Li and Y. Feng, "Carbon Nanotubes for Supercapacitor," Nanoscale Research Letters, Vol. 5, No. 3, 2010, pp. 654-668. doi:10.1007/s11671-009-9508-2

[9] Y. Huang, J. Liang and Y. Chen, "An Overview of the Applications of Graphene-Based Materials in Supercapacitors," Small, Vol. 8, No. 12, 2012, pp. 1805-1834. doi:10.1002/smll.201102635

[10] L. Yuan, X.-H. Lu, X. Xiao, T. Zhai, J. Dai, F. Zhang, et al., "Flexible Solid-State Supercapacitors Based on Carbon Nanoparticles $/ \mathrm{MnO}_{2}$ Nanorods Hybrid Structure," ACS Nano, Vol. 6, No. 1, 2012, pp. 656-661. doi: $10.1021 / \mathrm{nn} 2041279$

[11] M. Hughes, G. Z. Chen, M. S. P. Shaffer, D. J. Fray and A. H. Windle, "Electrochemical Capacitance of a Nanoporous Composite of Carbon Nanotubes and Polypyrrole," Chemistry of Materials, Vol. 14, No. 4, 2002, pp. 1610-1613. doi: $10.1021 / \mathrm{cm} 010744 \mathrm{r}$

[12] K. Zhang, L. L. Zhang, X. S. Zhao and J. Wu, "Graphene/ Polyaniline Nanofiber Composites as Supercapacitor Electrodes," Chemistry of Materials, Vol. 22, No. 4, 2010, pp. 1392-1401. doi:10.1021/cm902876u

[13] H. Wang, Q. Hao, X. Yang, L. Lu and X. Wang, "A Nanostructured Graphene/Polyaniline Hybrid Material for Supercapacitors," Nanoscale, Vol. 2, No. 10, 2010, p. 2164. doi:10.1039/c0nr00224k

[14] S. Bose, N. H. Kim, T. Kuila, K. Lau and J. H. Lee, "Electrochemical Performance of a Graphene-Polypyrrole Nanocomposite as a Supercapacitor Electrode," Nano-Technology, Vol. 22, No. 29, 2011, Article ID: 295202. doi:10.1088/0957-4484/22/29/295202

[15] Q. Wu, Y. Xu, Z. Yao, A. Liu and G. Shi, "Supercapacitors Based on Flexible Graphene/Polyaniline Nanofiber Composite Films," ACS Nano, Vol. 4, No. 4, 2010, pp. 1963-1970. doi:10.1021/nn1000035

[16] H. Gomez, M. K. Ram, F. Alvi, P. Villalba, E. (Lee) Stefanakos and A. Kumar, "Graphene-Conducting Polymer Nanocomposite as Novel Electrode for Supercapacitors," Journal of Power Sources, Vol. 196, No. 8, 2011, pp. 4102-4108. doi:10.1016/j.jpowsour.2010.11.002

[17] F. Alvi, M. K. Ram, P. A. Basnayaka, E. Stefanakos, Y. Goswami and A. Kumar, "Graphenepolyethylenedioxythiophene Conducting Polymer Nanocomposite Based Supercapacitor," Electrochimica Acta, Vol. 56, No. 25, 2011, pp. 9406-9412.

[18] F. Alvi, P. A. Basnayaka, M. K. Ram, H. Gomez, E. Stefanako, Y. Goswami and A. Kumar, "Graphene-Polythiophene Nanocomposite as Novel Supercapacitor Electrode Material," Journal of New Materials for Electrochemical Systems, Vol. 15, No. 2, 2012, pp. 89-95.

[19] F. Alvi, M. K. Ram, P. Basnayaka, E. Stefanakos, A. Hoff and A. Kumar, "Novel Graphene-Conducting Polythiophenes Materials for Applications in the Electrochemical Supercapacitors," ECS Meeting, Vol. 1, No. 11, 2011, p. 596.

[20] D. Zhang, X. Zhang, Y. Chen, P. Yu, C. Wang and Y. Ma, "Enhanced Capacitance and Rate Capability of Grphene/ Polypyrrole Composite as Electrode Material for Supercapacitors," Journal of Power Sources, Vol. 196, No. 14, 2011, pp. 5990-5996. doi:10.1016/j.jpowsour.2011.02.090

[21] S. Biswas and L.T. Drzal, "Multilayered Nanoarchitecture of Graphene Nanosheets and Polypyrrole Nanowires for High Performance Supercapacitor Electrodes," Chemistry of Materials, Vol. 22, No. 20, 2010, pp. 5667-5671. doi: $10.1021 / \mathrm{cm} 101132 \mathrm{~g}$

[22] C. Niu, E. K. Sichel, R. Hoch, D. Moy and H. Tennent, "High Power Electrochemical Capacitors Based on Car- 
bon Nanotube Electrodes," Applied Physics Letters, Vol. 70, No. 11, 1997, pp. 1480-1482. doi:10.1063/1.118568

[23] P. L. Taberna, P. Simon and J. F. Fauvarque, "Electrochemical Characteristics and Impedance Spectroscopy Studies of Carbon-Carbon Supercapacitors," Journal of the Electrochemical Society, Vol. 150, No. 3, 2003, pp. A292-A300. doi:10.1149/1.1543948

[24] J. Gamby, P. Taberna, P. Simon and J. F. Fauvarque, "Studies and Characterization of Various Activated Carbons Used for Carbon/Carbon Supercapacitors," Journal of Power Sources, Vol. 101, No. 1, 2001, pp. 109-116.

[25] Y. Zhu, S. Murali, M. D. Stoller, K. J. Ganesh, W. Cai, P. J. Ferreira, A. Pirkle, R. M. Wallace, K. A. Cychosz, M. Thommes, D. Su, E. A. Stach and R. S. Ruoff, "CarbonBased Supercapacitors Produced by Activation of Gra- phene," Science, Vol. 332, No. 6037, 2011, pp. 1537 1541. doi:10.1126/science. 1200770

[26] V. V. Karambelkar and J. D. Ekhe, "High Yield Polypyrrole: A Novel Approach to Synthesis and Characterization," Journal of Material Science, Vol. 46, No. 16, 2011, pp. 5324-5331. doi:10.1007/s10853-011-5470-3

[27] A. C. Ferrari, J. C. Meyer, V. Scardaci, C. Casiraghi, M. Lazzeri, F. Mauri, S. Piscanec, D. Jiang, K. S. Novoselov, S. Roth and A. K. Geim, "Raman Spectrum of Graphene and Graphene Layers," Physical Review Letters, Vol. 97, No. 18, 2006, Article ID: 187401 doi:10.1103/PhysRevLett.97.187401

[28] V. Daniel, "Dielectric Relaxation," Academic Press, London and New York, 1967. 